\title{
QUASI-PARTICLE BASES OF PRINCIPAL SUBSPACES OF THE AFFINE LIE ALGEBRA OF TYPE $G_{2}^{(1)}$
}

\author{
MARIJANA BUTORAC \\ University of Rijeka, Croatia
}

ABstract. The aim of this work is to construct the quasi-particle basis of principal subspace of standard module of highest weight $k \Lambda_{0}$ of level $k \geq 1$ of affine Lie algebra of type $G_{2}^{(1)}$ by means of which we obtain the basis of principal subspace of generalized Verma module.

\section{INTRODUCTION}

Principal subspaces of standard modules of affine Lie algebras $A_{1}^{(1)}$ were first introduced by B. L. Feigin and A. V. Stoyanovsky in [16]. Motivated by the work of J. Lepowsky and M. Primc ([26]), Feigin and Stoyanovsky related characters of principal subspaces with Rogers-Ramanujan type identities. This connection was further studied by many authors, in particular in [3], [6, 7], [8-11], [12], [13,14], [18], [24], [28, 29], [30,31] and others. More recently, Slaven Kožić in $[22,23]$ showed that character formulas for level 1 principal subspaces associated with the integrable highest weight module of quantum affine algebra $U_{q}\left(\widehat{\mathfrak{s l}_{2}}\right)$ coincide with the character formulas found in [16].

In [18], G. Georgiev constructed bases for principal subspaces of certain standard $A_{l}^{(1)}$-modules by using monomials of certain vertex operator coefficients corresponding to simple roots of $A_{l}$, the so-called quasi-particles (cf.

2010 Mathematics Subject Classification. 17B67, 17B69, 05A19.

Key words and phrases. Affine Lie algebras, vertex operator algebras, principal subspaces, quasi-particle bases.

This work has been supported in part by the Croatian Science Foundation under the project 2634., by the Croatian Scientific Centre of Excellence QuantiXLie and by University of Rijeka research grant 13.14.1.2.02. 
[16]), from which were easily obtained the Rogers-Ramanujan type character formulas. In [4] and [5] we extended Georgiev's construction of quasiparticle bases for principal subspaces of standard module $L\left(k \Lambda_{0}\right)$ and generalized Verma module $N\left(k \Lambda_{0}\right)$ of highest weight $k \Lambda_{0}, k \in \mathbb{N}$ for affine Lie algebras of type $B_{l}^{(1)}$ and $C_{l}^{(1)}, l \geq 2$. As a consequence we proved two new series of Rogers-Ramanujan type identities obtained from the characters of principal subspaces of generalized Verma module.

In this note we construct quasi-particle bases of principal subspaces of generalized Verma module $N\left(k \Lambda_{0}\right)$ and its irreducible quotient in the case of affine Lie algebra of type $G_{2}^{(1)}$. Two main steps in the construction are similar to the case of $B_{2}^{(1)}$. First step is to find relations among quasi-particles from which follow the spanning set of principal subspaces and the second step is to prove that the spanning set is linearly independent by induction on the linear order on quasi-particles. The main differences with the case of $B_{2}^{(1)}$ are relations which describe the interaction of quasi-particles associated to different simple roots and operators which we use in the proof of linear independence, since we don't have a simple current operator as in the proof of independence for $B_{2}^{(1)}$.

To state our main results, denote by $W_{L\left(k \Lambda_{0}\right)}$ the principal subspace of level $k$ standard module and by ch $W_{L\left(k \Lambda_{0}\right)}$ the character of $W_{L\left(k \Lambda_{0}\right)}$ and by $W_{N\left(k \Lambda_{0}\right)}$ the principal subspace of generalized Verma module $N\left(k \Lambda_{0}\right)$. Our result states:

\section{THEOREM 1.1.}

$\operatorname{ch} W_{L\left(k \Lambda_{0}\right)}$

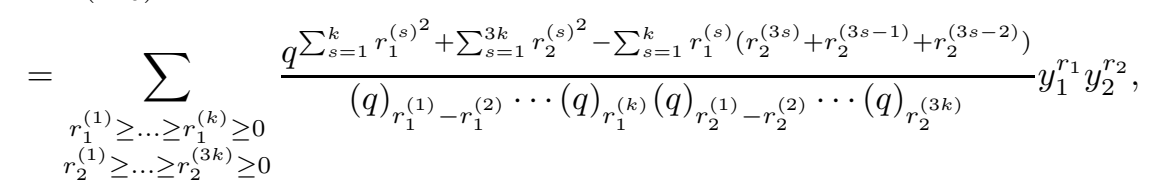

where $r_{1}=\sum_{s=1}^{k} r_{1}^{(s)}$ and $r_{2}=\sum_{s=1}^{3 k} r_{2}^{(s)}$.

This new fermionic formula which follows directly from quasi-particle basis of $W_{L\left(k \Lambda_{0}\right)}$ is related to the study of parafermionic Rogers-Ramanujan type characters $([19])$.

We use quasi-particle bases of $W_{L\left(k \Lambda_{0}\right)}$ in the construction of quasi-particles bases of principal subspace $W_{N\left(k \Lambda_{0}\right)}$ of generalized Verma module, from which follows a generalization of Euler-Cauchy identity. 


\section{THEOREM 1.2 .}

(1.1)

$$
\begin{aligned}
& \prod_{m>0} \frac{1}{\left(1-q^{m} y_{1}\right)} \frac{1}{\left(1-q^{m} y_{2}\right)} \frac{1}{\left(1-q^{m} y_{1} y_{2}\right)} \\
& \frac{1}{\left(1-q^{m} y_{1} y_{2}^{2}\right)} \frac{1}{\left(1-q^{m} y_{1} y_{2}^{3}\right)} \frac{1}{\left(1-q^{m} y_{1}^{2} y_{2}^{3}\right)}
\end{aligned}
$$

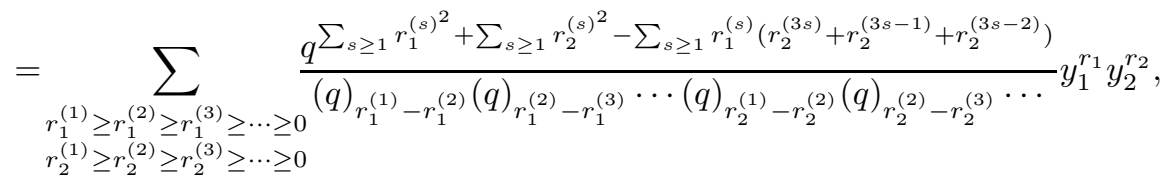

where $r_{1}=\sum_{s>1} r_{1}^{(s)}$ and $r_{2}=\sum_{s>1} r_{2}^{(s)}$. The sum on the right side of (1.1) is over all descending infinite sequences of non-negative integers with finite support.

\section{PRINCIPAL SUBSPACES}

Let $\mathfrak{g}$ be a complex simple Lie algebra of type $G_{2}$ with a triangular decomposition $\mathfrak{g}=\mathfrak{n}_{-} \oplus \mathfrak{h} \oplus \mathfrak{n}_{+}$, with the basis $\Pi=\left\{\alpha_{1}=\frac{1}{\sqrt{3}}\left(-2 \epsilon_{1}+\epsilon_{2}+\epsilon_{3}\right), \alpha_{2}=\right.$ $\left.\frac{1}{\sqrt{3}}\left(\epsilon_{1}-\epsilon_{2}\right)\right\}$ of the root system $R$ and the corresponding set of fundamental weights $\left\{\omega_{1}=2 \alpha_{1}+3 \alpha_{2}, \omega_{2}=\alpha_{1}+2 \alpha_{2}\right\}$, where $\epsilon_{1}, \epsilon_{2}, \epsilon_{3}$ are vectors of the standard basis of $\mathbb{R}^{3}$. Denote by $\theta=\frac{1}{\sqrt{3}}\left(-\epsilon_{1}-\epsilon_{2}+2 \epsilon_{3}\right)$ the highest root and assume that all long roots $\alpha \in R$ are normalized by the condition $\langle\alpha, \alpha\rangle=2$, where $\langle\cdot, \cdot\rangle$ denotes the invariant nondegenarate bilinear form on $\mathfrak{g}$, which induces a bilinear form on $\mathfrak{h}^{*}$. Denote by $Q$ the root lattice and by $P$ the weight lattice of $\mathfrak{g}$. Then, $P=Q$. For later use we fix root vectors

$$
\begin{aligned}
& x_{\alpha_{1}+\alpha_{2}}=\left[x_{\alpha_{2}}, x_{\alpha_{1}}\right], x_{\alpha_{1}+2 \alpha_{2}}=\left[x_{\alpha_{2}}, x_{\alpha_{1}+\alpha_{2}}\right], \\
& x_{\alpha_{1}+3 \alpha_{2}}=\left[x_{\alpha_{2}}, x_{\alpha_{1}+2 \alpha_{2}}\right], x_{2 \alpha_{1}+3 \alpha_{2}}=\left[x_{\alpha_{1}}, x_{\alpha_{1}+3 \alpha_{2}}\right] .
\end{aligned}
$$

Let $\widetilde{\mathfrak{g}}$ be the associated affine Lie algebra

$$
\widetilde{\mathfrak{g}}=\widehat{\mathfrak{g}} \oplus \mathbb{C} d, \quad \widehat{\mathfrak{g}}=\mathfrak{g} \otimes \mathbb{C}\left[t, t^{-1}\right] \oplus \mathbb{C} c,
$$

with commutation relations

$$
\begin{gathered}
{\left[x\left(j_{1}\right), y\left(j_{2}\right)\right]=[x, y]\left(j_{1}+j_{2}\right)+\langle x, y\rangle j_{1} \delta_{j_{1}+j_{2}, 0} c} \\
{[c, \mathfrak{\mathfrak { g }}]=0, \quad[d, x(j)]=j x(j),}
\end{gathered}
$$

where $x(j)=x \otimes t^{j}$ for $x, y \in \mathfrak{g}, j, j_{1}, j_{2} \in \mathbb{Z},($ cf. [21]). We consider $\widetilde{\mathfrak{g}}$ subalgebras

$$
\begin{gathered}
\mathcal{L}\left(\mathfrak{n}_{+}\right)=\mathfrak{n}_{+} \otimes \mathbb{C}\left[t, t^{-1}\right], \\
\mathcal{L}\left(\mathfrak{n}_{+}\right)_{\geq 0}=\mathfrak{n}_{+} \otimes \mathbb{C}[t], \quad \mathcal{L}\left(\mathfrak{n}_{+}\right)_{<0}=\mathfrak{n}_{+} \otimes t^{-1} \mathbb{C}\left[t^{-1}\right]
\end{gathered}
$$

and

$$
\mathcal{L}\left(\mathfrak{n}_{\alpha}\right)=\mathfrak{n}_{\alpha} \otimes \mathbb{C}\left[t, t^{-1}\right]
$$


where

$$
\mathfrak{n}_{\alpha}=\mathbb{C} x_{\alpha}
$$

are one-dimensional $\mathfrak{g}$-subalgebras generated with root vectors $x_{\alpha}, \alpha \in R$.

We extend our form $\langle\cdot, \cdot\rangle$ to $\widetilde{\mathfrak{h}}=\mathfrak{h} \oplus \mathbb{C} c \oplus \mathbb{C} d$. The set of simple roots of $\widetilde{\mathfrak{g}}$ is $\left\{\alpha_{0}, \alpha_{1}, \alpha_{2}\right\}$ and $\left\{\Lambda_{0}, \Lambda_{1}, \Lambda_{2}\right\}$ is the set of fundamental weights. Denote by $L\left(\Lambda_{0}\right)$ a standard (i.e. integrable highest weight) $\widetilde{\mathfrak{g}}$-module of level 1 with the highest weight vector $v_{L\left(\Lambda_{0}\right)}$.

Fix $k \in \mathbb{N}$. Denote by $N\left(k \Lambda_{0}\right)$ the generalized Verma module and by $L\left(k \Lambda_{0}\right)$ its irreducible quotient. The induced $\widetilde{\mathfrak{g}}$-module $N\left(k \Lambda_{0}\right)$ is defined as

$$
N\left(k \Lambda_{0}\right)=U(\widehat{\mathfrak{g}}) \otimes_{U\left(\widehat{\mathfrak{g}}_{\geq 0}\right)} \mathbb{C} v_{N\left(k \Lambda_{0}\right)},
$$

where $\widehat{\mathfrak{g}}_{\geq 0}=\bigoplus_{n \geq 0} \mathfrak{g} \otimes t^{n} \oplus \mathbb{C} c$ and $\mathbb{C} v_{N\left(k \Lambda_{0}\right)}$ is 1-dimensional $\widehat{\mathfrak{g}}_{\geq 0}$-module, such that

$$
c v_{N\left(k \Lambda_{0}\right)}=k v_{N\left(k \Lambda_{0}\right)}, \quad d v_{N\left(k \Lambda_{0}\right)}=0, \quad\left(\mathfrak{g} \otimes t^{j}\right) v_{N\left(k \Lambda_{0}\right)}=0, \quad j \geq 0 .
$$

Set

$$
v_{N\left(k \Lambda_{0}\right)}=1 \otimes v_{N\left(k \Lambda_{0}\right)} .
$$

The generalized Verma module has a structure of a vertex operator algebra, as its irreducible quotient $L\left(k \Lambda_{0}\right)$ and all the level $\mathrm{k}$ standard modules are modules for vertex operator algebra $L\left(k \Lambda_{0}\right)$. The vertex operator map is determined by

$$
Y\left(x(-1) v_{N\left(k \Lambda_{0}\right)}, z\right)=\sum_{m \in \mathbb{Z}} x(m) z^{-m-1}=x(z)
$$

for $x \in \mathfrak{g}$ (cf. [25]). We will use the commutator formula among vertex operators:

$$
\begin{aligned}
& {\left[Y\left(x_{\alpha}(-1) v_{N\left(k \Lambda_{0}\right)}, z_{1}\right), Y\left(x_{\beta}(-1)^{r} v_{N\left(k \Lambda_{0}\right)}, z_{2}\right)\right]} \\
& \quad=\sum_{j \geq 0} \frac{(-1)^{j}}{j !}\left(\frac{d}{d z_{1}}\right)^{j} z_{2}^{-1} \delta\left(\frac{z_{1}}{z_{2}}\right) Y\left(x_{\alpha}(j) x_{\beta}(-1)^{r} v_{N\left(k \Lambda_{0}\right)}, z_{2}\right),
\end{aligned}
$$

where $\alpha, \beta \in R$, (cf. [17]).

Denote by $v_{L\left(k \Lambda_{0}\right)}$ the highest weight vector of $L\left(k \Lambda_{0}\right)$. We define a principal subspace $W_{L\left(k \Lambda_{0}\right)}$ of $L\left(k \Lambda_{0}\right)$ (see $\left.[16,18]\right)$ as

$$
W_{L\left(k \Lambda_{0}\right)}=U\left(\mathcal{L}\left(\mathfrak{n}_{+}\right)\right) v_{L\left(k \Lambda_{0}\right)}
$$

and the principal subspace $W_{N\left(k \Lambda_{0}\right)}$ of the generalized Verma module $N\left(k \Lambda_{0}\right)$ as

$$
W_{N\left(k \Lambda_{0}\right)}=U\left(\mathcal{L}\left(\mathfrak{n}_{+}\right)\right) v_{N\left(k \Lambda_{0}\right)} .
$$

Note that the map

$$
f: U\left(\mathcal{L}\left(\mathfrak{n}_{+}\right)_{<0}\right) \rightarrow W_{N\left(k \Lambda_{0}\right)}, \quad f(b)=b v_{N\left(k \Lambda_{0}\right)}
$$

is an isomorphism of $\mathcal{L}\left(\mathfrak{n}_{+}\right)_{<0}$-modules. If we order basis elements of $\mathfrak{n}_{+}$

$$
\left\{x_{\alpha_{1}}, x_{\alpha_{2}}, x_{\alpha_{1}+\alpha_{2}}, x_{\alpha_{1}+2 \alpha_{2}}, x_{\alpha_{1}+3 \alpha_{2}}, x_{2 \alpha_{1}+3 \alpha_{2}}\right\}
$$


in the following way:

$$
x_{\alpha_{2}}<x_{\alpha_{1}}<x_{\alpha_{1}+\alpha_{2}}<x_{\alpha_{1}+2 \alpha_{2}}<x_{\alpha_{1}+3 \alpha_{2}}<x_{2 \alpha_{1}+3 \alpha_{2}}
$$

and basis elements of $\mathcal{L}\left(\mathfrak{n}_{+}\right)_{<0}$

$$
\left\{x_{\alpha}(m): \alpha \in R_{+}, m<0\right\}
$$

as:

$$
x(m) \leq y\left(m^{\prime}\right) \Leftrightarrow x<y \text { or } x=y \text { and } m<m^{\prime},
$$

then from the Poincaré-Birkhoff-Witt theorem follows that vectors

$$
\begin{aligned}
& x_{\alpha_{2}}\left(m_{1}^{1}\right) \cdots x_{\alpha_{2}}\left(m_{1}^{s_{1}}\right) x_{\alpha_{1}}\left(m_{2}^{1}\right) \cdots x_{\alpha_{1}}\left(m_{2}^{s_{2}}\right) \\
& \quad \cdots x_{2 \alpha_{1}+3 \alpha_{2}}\left(m_{6}^{1}\right) \cdots x_{2 \alpha_{1}+3 \alpha_{2}}\left(m_{6}^{s_{6}}\right) v_{N\left(k \Lambda_{0}\right)},
\end{aligned}
$$

where $m_{i}^{1} \leq \cdots \leq m_{i}^{s_{i}}<0, s_{i} \geq 0,1 \leq i \leq 6$, form a basis of a vector space $W_{N\left(k \Lambda_{0}\right)}$.

In next sections, we construct bases of principal subspaces $W_{L\left(k \Lambda_{0}\right)}$ and $W_{N\left(k \Lambda_{0}\right)}$ in terms of certain coefficients of vertex operators corresponding to vectors $x_{\alpha_{i}}(-1)^{r} v_{L\left(k \Lambda_{0}\right)}$ (and $\left.x_{\alpha_{i}}(-1)^{r} v_{N\left(k \Lambda_{0}\right)}\right)$, where $r \geq 1$ and $\alpha_{i} \in \Pi$.

First, we choose a special subspace of $U\left(\mathcal{L}\left(\mathfrak{n}_{+}\right)\right)$

$$
U=U\left(\mathcal{L}\left(\mathfrak{n}_{\alpha_{2}}\right)\right) U\left(\mathcal{L}\left(\mathfrak{n}_{\alpha_{1}}\right)\right) .
$$

It is easy to see that principal subspaces are generated by operators in $U$ acting on the highest weight vectors $v_{L\left(k \Lambda_{0}\right)}$ and $v_{N\left(k \Lambda_{0}\right)}$ (see Lemma 3.1 in $[18])$.

\section{QuASI-PARTIClE BASES OF PRINCIPAL SUBSPACES}

We start this section with introducing all necessary notions and facts needed in the construction of quasi-particle bases of principal subspaces. Some terms and labels which we use, but are not mentioned, are the same as in our previous work, therefore, for more details we refer to $[4,5]$ and also to [18].

3.1. Quasi-particle monomials. For given $i \in\{1,2\}, r \in \mathbb{N}$ and $m \in \mathbb{Z}$ define a quasi-particle of color $i$, charge $r$ and energy $-m$ by

$$
x_{r \alpha_{i}}(m)=\operatorname{Res}_{z}\left\{z^{m+r-1} x_{r \alpha_{i}}(z)\right\},
$$

where $x_{r \alpha_{i}}(z)$ is a vertex operator

$$
x_{r \alpha_{i}}(z):=x_{\alpha_{i}}(z)^{r}=Y\left(\left(x_{\alpha_{i}}(-1)\right)^{r} v_{L\left(k \Lambda_{0}\right)}, z\right) .
$$

$x_{r \alpha_{i}}(z)$ is the generating function of quasi-particles of color $i$ and charge $r$.

Denote by $b\left(\alpha_{i}\right)$ the monochromatic quasi-particle monomial, that is the product of quasi-particles of the same color $i$. We say that monomial $b$ "colored" with more colors is a polychromatic monomial. As in the case of $B_{2}^{(1)}$, our basis monomials will be "colored" with two colors $i=1,2$ and our monomials will have the form

$$
b=b\left(\alpha_{2}\right) b\left(\alpha_{1}\right) .
$$


For monomial

$$
\begin{aligned}
b\left(\alpha_{2}\right) b\left(\alpha_{1}\right)= & x_{n_{r_{2}^{(1)}, 2} \alpha_{2}}\left(m_{r_{2}^{(1)}, 2}\right) \cdots x_{n_{1,2} \alpha_{2}}\left(m_{1,2}\right) \\
& x_{n_{r_{1}^{(1)}, 1} \alpha_{1}}\left(m_{r_{1}^{(1)}, 1}\right) \cdots x_{n_{1,1} \alpha_{1}}\left(m_{1,1}\right)
\end{aligned}
$$

we will say it is of charge-type

$$
\mathcal{R}^{\prime}=\left(n_{r_{2}^{(1)}, 2}, \ldots, n_{1,2} ; n_{r_{1}^{(1)}, 1}, \ldots, n_{1,1}\right),
$$

where

$$
0 \leq n_{r_{i}^{(1)}, i} \leq \ldots \leq n_{1, i}
$$

dual-charge-type

$$
\mathcal{R}=\left(r_{2}^{(1)}, \ldots, r_{2}^{\left(s_{2}\right)} ; r_{1}^{(1)}, \ldots, r_{1}^{\left(s_{1}\right)}\right)
$$

where

$$
r_{i}^{(1)} \geq r_{i}^{(2)} \geq \ldots \geq r_{i}^{\left(s_{i}\right)} \geq 0
$$

and color-type

$$
\left(r_{2}, r_{1}\right)
$$

where

$$
r_{i}=\sum_{p=1}^{r_{i}^{(1)}} n_{p, i}=\sum_{t=1}^{s_{i}} r_{i}^{(t)} \text { and } s_{i} \in \mathbb{N},
$$

(cf. [4, 5, 18]) if for every color $\mathcal{R}$ and $\mathcal{R}^{\prime}$ are mutually conjugate partitions of $r_{i}$ (cf. [1]). We use the same terminology for the products of generating functions.

We assume that all monomial factors are sorted so that energies of quasiparticles of the same color and the same charge form an increasing sequence of integers from right to left. We compare charge-type $\mathcal{R}^{\prime}$ and $\overline{\mathcal{R}^{\prime}}$, where $\overline{\mathcal{R}^{\prime}}=$ $\left(\bar{n}_{\bar{r}_{2}^{(1)}, 2}, \ldots, \bar{n}_{1,1}\right)$, so that we compare their charges from right to left, i.e. we write $\mathcal{R}^{\prime}<\overline{\mathcal{R}^{\prime}}$ if there is $u \in \mathbb{N}$, such that $n_{1, i}=\bar{n}_{1, i}, n_{2, i}=\bar{n}_{2, i}, \ldots, n_{u-1, i}=$ $\bar{n}_{u-1, i}$, and $u=\bar{r}_{i}^{(1)}+1$ or $n_{u, i}<\bar{n}_{u, i}$.

We compare two monomials $b$ and $\bar{b}$ by comparing first their chargetypes $\mathcal{R}^{\prime}$ and $\overline{\mathcal{R}^{\prime}}$ and then their sequences of energies $\left(m_{r_{2}^{(1)}, 2}, \ldots, m_{1,1}\right)$ and $\left(\bar{m}_{\bar{r}_{2}^{(1)}, 2}, \ldots, \bar{m}_{1,1}\right)$ (in a similar way as charge-types, again starting from color $i=1)$ :

$$
b<\bar{b} \text { if }\left\{\begin{array}{l}
\mathcal{R}^{\prime}<\overline{\mathcal{R}^{\prime}}, \\
\mathcal{R}^{\prime}=\overline{\mathcal{R}^{\prime}}
\end{array} \text { and }\left(m_{r_{2}^{(1)}, 2}, \ldots, m_{1,1}\right)<\left(\bar{m}_{\bar{r}_{2}^{(1)}, 2}, \ldots, \bar{m}_{1,1}\right) .\right.
$$


3.2. Relations among quasi-particles. On a standard module $L\left(k \Lambda_{0}\right)$, we have vertex operator algebra relations

$$
\begin{gathered}
x_{(k+1) \alpha_{1}}(z)=0, \\
x_{(3 k+1) \alpha_{2}}(z)=0, \\
x_{n \alpha_{i}}(z) v_{L\left(k \Lambda_{0}\right)} \in W_{L\left(k \Lambda_{0}\right)}[[z]],
\end{gathered}
$$

and

$$
x_{n \alpha_{i}}(m) v_{L\left(k \Lambda_{0}\right)}=0, \text { for } m>-n,
$$

when $n \leq k$ for $i=1$ and $n \leq 3 k$ for $i=2$, (see [25,27]).

In reducing the set $U v_{L\left(k \Lambda_{0}\right)}$ to the spanning set we use relations for a sequence of monochromatic monomial vectors (see Lemma 2.2.1 in [4], or $[20,18,15])$

$$
\begin{gathered}
x_{n \alpha_{i}}(m) x_{n^{\prime} \alpha_{i}}\left(m^{\prime}\right) v_{L\left(k \Lambda_{0}\right)}, x_{n \alpha_{i}}(m-1) x_{n^{\prime} \alpha_{i}}\left(m^{\prime}+1\right) v_{L\left(k \Lambda_{0}\right)}, \ldots \\
\ldots, x_{n \alpha_{i}}(m-2 n+1) x_{n^{\prime} \alpha_{i}}\left(m^{\prime}+2 n-1\right) v_{L\left(k \Lambda_{0}\right)},
\end{gathered}
$$

colored with color $i$ and with charge-type $\left(n, n^{\prime}\right)$, where $n<n^{\prime}$, which we express as a (finite) linear combination of monomial vectors

$$
x_{n \alpha_{i}}(j) x_{n^{\prime} \alpha_{i}}\left(j^{\prime}\right) v_{L\left(k \Lambda_{0}\right)} \text { such that } j \leq m-2 n \text { and } j^{\prime} \geq m^{\prime}+2 n
$$

and monomial vectors with a factor quasi-particle $x_{\left(n^{\prime}+1\right) \alpha_{i}}\left(j_{1}\right), j_{1} \in \mathbb{Z}$.

In the case when $n=n^{\prime}$ monomials

$$
x_{n \alpha_{i}}(m) x_{n \alpha_{i}}\left(m^{\prime}\right) \text { with } m^{\prime}-2 n<m \leq m^{\prime}
$$

can be expressed as a linear combination of monomials

$$
x_{n \alpha_{i}}(j) x_{n \alpha_{i}}\left(j^{\prime}\right) \text { with } j \leq j^{\prime}-2 n
$$

and monomials with quasi-particle $x_{(n+1) \alpha_{i}}\left(j_{1}\right), j_{1} \in \mathbb{Z}$ (see Corollary 2.2.2 in $[4]$, or $[20,18,15])$.

Next, we consider products of quasi-particles colored with different colors. First, from commutation formulas (2.1) and (2.2) and induction on $n, n^{\prime} \in \mathbb{N}$ follows

LEMMA 3.1. Let $n \leq 3 k, n^{\prime} \leq k$ be fixed. We have:

a)

$$
\begin{aligned}
x_{\alpha_{1}}(0) x_{\alpha_{2}}^{n}(-1) v_{L\left(k \Lambda_{0}\right)}= & -n x_{\alpha_{2}}^{n-1}(-1) x_{\alpha_{1}+\alpha_{2}}(-1) v_{L\left(k \Lambda_{0}\right)} \\
& +\left(\begin{array}{c}
n \\
2
\end{array}\right) x_{\alpha_{2}}^{n-2}(-1) x_{\alpha_{1}+2 \alpha_{2}}(-2) v_{L\left(k \Lambda_{0}\right)} \\
& -\left(\begin{array}{l}
n \\
3
\end{array}\right) x_{\alpha_{2}}^{n-3}(-1) x_{\alpha_{1}+3 \alpha_{2}}(-3) v_{L\left(k \Lambda_{0}\right)}
\end{aligned}
$$


b)

$$
\begin{aligned}
x_{\alpha_{1}}(1) x_{\alpha_{2}}^{n}(-1) v_{L\left(k \Lambda_{0}\right)}= & \left(\begin{array}{l}
n \\
2
\end{array}\right) x_{\alpha_{2}}^{n-2}(-1) x_{\alpha_{1}+2 \alpha_{2}}(-1) v_{L\left(k \Lambda_{0}\right)} \\
& -\left(\begin{array}{c}
n \\
3
\end{array}\right) x_{\alpha_{2}}^{n-3}(-1) x_{\alpha_{1}+3 \alpha_{2}}(-2) v_{L\left(k \Lambda_{0}\right)} ;
\end{aligned}
$$

c) $x_{\alpha_{1}}(2) x_{\alpha_{2}}^{n}(-1) v_{L\left(k \Lambda_{0}\right)}=-\left(\begin{array}{l}n \\ 3\end{array}\right) x_{\alpha_{2}}^{n-3}(-1) x_{\alpha_{1}+3 \alpha_{2}}(-1) v_{L\left(k \Lambda_{0}\right)}$;

d) $x_{\alpha_{1}}(j) x_{\alpha_{2}}^{n}(-1) v_{L\left(k \Lambda_{0}\right)}=0$, where $j \geq 3$;

e) $x_{\alpha_{2}}(0) x_{\alpha_{1}}^{n^{\prime}}(-1) v_{L\left(k \Lambda_{0}\right)}=n^{\prime} x_{\alpha_{1}}^{n^{\prime}-1} x_{\alpha_{1}+\alpha_{2}}(-1) v_{L\left(k \Lambda_{0}\right)}$;

f) $x_{\alpha_{2}}(j) x_{\alpha_{1}}^{n^{\prime}}(-1) v_{L\left(k \Lambda_{0}\right)}=0$, where $j \geq 1$.

The previous lemma implies relation among quasi-particles of different colors:

LEMMA 3.2. Let $n_{1} \leq k, n_{2} \leq 3 k$. One has

$$
\begin{aligned}
& \left(z_{1}-z_{2}\right)^{\min \left\{3 n_{1}, n_{2}\right\}} x_{n_{1} \alpha_{1}}\left(z_{1}\right) x_{n_{2} \alpha_{2}}\left(z_{2}\right) \\
& \quad=\left(z_{1}-z_{2}\right)^{\min \left\{3 n_{1}, n_{2}\right\}} x_{n_{2} \alpha_{2}}\left(z_{2}\right) x_{n_{1} \alpha_{1}}\left(z_{1}\right) .
\end{aligned}
$$

Proof. Note, that from commutator formula for vertex operators (2.3), statements a), b), c) and d) of Lemma 3.1 and properties of $\delta$-function we have

$$
\left(z_{1}-z_{2}\right)^{3} x_{\alpha_{1}}\left(z_{1}\right) x_{n_{2} \alpha_{2}}\left(z_{2}\right)=\left(z_{1}-z_{2}\right)^{3} x_{n_{2} \alpha_{2}}\left(z_{2}\right) x_{\alpha_{1}}\left(z_{1}\right) .
$$

In a similar way, using e) and f) parts of Lemma 3.1 we have

$$
\left(z_{1}-z_{2}\right) x_{n_{1} \alpha_{1}}\left(z_{1}\right) x_{\alpha_{2}}\left(z_{2}\right)=\left(z_{1}-z_{2}\right) x_{\alpha_{2}}\left(z_{2}\right) x_{n_{1} \alpha_{1}}\left(z_{1}\right) .
$$

Now, from (3.8) and (3.9) follows the lemma.

By using derived relations we can define the set of quasi-particle monomials which generate our bases (acting on the highest weight vectors)

$$
\begin{aligned}
& B_{W_{L\left(k \Lambda_{0}\right)}}=\bigcup_{\substack{n_{r_{1}^{(1)}, 1} \leq \ldots \leq n_{1,1} \leq k \\
n_{r_{2}^{(1)}, 2} \leq \ldots \leq n_{1,2} \leq 3 k}}\left(\begin{array}{ll}
\text { or, equivalently, } & \bigcup^{r_{1}^{(1)} \geq \cdots \geq r_{1}^{(k)} \geq 0} \\
r_{2}^{(1)} \geq \cdots \geq r_{2}^{(3 k)} \geq 0
\end{array}\right) \\
& \left\{b=b\left(\alpha_{2}\right) b\left(\alpha_{1}\right)\right. \\
& =x_{n_{r_{2}(1), 2} \alpha_{2}}\left(m_{r_{2}^{(1)}, 2}\right) \cdots x_{n_{1,2} \alpha_{2}}\left(m_{1,2}\right) x_{n_{r_{1}^{(1)}, 1} \alpha_{1}}\left(m_{r_{1}^{(1)}, 1}\right) \cdots x_{n_{1,1} \alpha_{1}}\left(m_{1,1}\right):
\end{aligned}
$$




$$
\left.\mid \begin{array}{l}
m_{p, 1} \leq-n_{p, 1}-\sum_{p>p^{\prime}>0} 2 \min \left\{n_{p, 1}, n_{p^{\prime}, 1}\right\}, 1 \leq p \leq r_{1}^{(1)} \\
m_{p+1,1} \leq m_{p, 1}-2 n_{p, 1} \text { if } n_{p+1,1}=n_{p, 1}, 1 \leq p \leq r_{1}^{(1)}-1 \\
m_{p, 2} \leq-n_{p, 2}+\sum_{q=1}^{r_{1}^{(1)}} \min \left\{3 n_{q, 1}, n_{p, 2}\right\}-\sum_{p>p^{\prime}>0} 2 \min \left\{n_{p, 2}, n_{p^{\prime}, 2}\right\} \\
\quad 1 \leq p \leq r_{2}^{(1)} ; \\
m_{p+1,2} \leq m_{p, 2}-2 n_{p, 2} \text { if } n_{p, 2}=n_{p+1,2}, 1 \leq p \leq r_{2}^{(1)}-1
\end{array}\right\}
$$

The condition on energies of quasi-particles colored with color $i=2$ contains a part which follows from relation (3.7). The other conditions on energies which follow from relations (3.1)-(3.6) are similar to difference conditions as in the case of $B_{2}^{(1)}$.

Now, we can state the Proposition 3.3, whose proof follows closely [18].

Proposition 3.3. The set

$$
\mathfrak{B}_{W_{L\left(k \Lambda_{0}\right)}}=\left\{b v_{L\left(k \Lambda_{0}\right)}: b \in B_{W_{L\left(k \Lambda_{0}\right)}}\right\}
$$

spans the principal subspace $W_{L\left(k \Lambda_{0}\right)}$.

In the rest of this section we consider the proof of linear independence of the set $\mathfrak{B}_{W_{L\left(k \Lambda_{0}\right)}}$. First, we introduce the properties of operators on a standard module level 1, which we will use in our proof.

3.3. Projection $\pi_{\mathcal{R}}$. Let $k>1$. We realize the principal subspace $W_{L\left(k \Lambda_{0}\right)}$ as a subspace of the tensor product $W_{L\left(\Lambda_{0}\right)}^{\otimes k} \subset L\left(\Lambda_{0}\right)^{\otimes k}$, where

$$
v_{L\left(k \Lambda_{0}\right)}=\underbrace{v_{L\left(\Lambda_{0}\right)} \otimes \cdots \otimes v_{L\left(\Lambda_{0}\right)}}_{k \text { factors }}
$$

is the highest weight vector.

For a chosen dual-charge-type

$$
\mathfrak{R}=\left(r_{2}^{(1)}, \ldots, r_{2}^{(3 k)} ; r_{1}^{(1)}, \ldots, r_{1}^{(k)}\right),
$$

denote by $\pi_{\mathfrak{R}}$ the projection of principal subspace $W_{L\left(k \Lambda_{0}\right)}$ to the subspace

$$
W_{L\left(\Lambda_{0}\right)\left(\mu_{2}^{(k)} ; r_{1}^{(k)}\right)} \otimes \cdots \otimes W_{L\left(\Lambda_{0}\right)\left(\mu_{2}^{(1)} ; r_{1}^{(1)}\right)},
$$

where $W_{L\left(\Lambda_{0}\right)\left(\mu_{2}^{(t)} ; r_{1}^{(t)}\right)}$ is a $\mathfrak{h}$-weight subspace of weight $\mu_{2}^{(t)} \alpha_{2}+r_{1}^{(t)} \alpha_{1} \in Q$ with

for every $1 \leq t \leq k$.

$$
\mu_{2}^{(t)}=r_{2}^{(3 t)}+r_{2}^{(3 t-1)}+r_{2}^{(3 t-2)}
$$

We shall denote by the same symbol $\pi_{\mathfrak{R}}$ the generalization of this projection to the space of formal series with coefficients in $W_{L\left(\Lambda_{0}\right)}^{\otimes k}$. Let

$$
x_{n_{2}^{(1), 2}} \alpha_{2}\left(z_{r_{2}^{(1)}, 2}\right) \cdots x_{n_{1,2} \alpha_{2}}\left(z_{1,2}\right) x_{n_{r_{1}^{(1)}, 1} \alpha_{1}}\left(z_{r_{1}^{(1)}, 1}\right) \cdots x_{n_{1,1} \alpha_{1}}\left(z_{1,1}\right)
$$

be a generating function of the chosen dual-charge-type $\mathfrak{R}$ and the corresponding charge-type $\mathfrak{R}^{\prime}$. Then, from relations (3.1) and (3.2) and definition 
of the action of Lie algebra on the modules, follows that the projection of the generating function (3.10) is

$$
\begin{aligned}
& \pi_{\Re} x_{n_{2}^{(1)}, \alpha_{2}}\left(z_{r_{2}^{(1)}, 2}\right) \cdots x_{n_{1,1} \alpha_{1}}\left(z_{1,1}\right) v_{L\left(k \Lambda_{0}\right)} \\
& =\mathrm{C} x_{n_{r_{2}^{(k k-2), 2}}^{(k)} \alpha_{2}}\left(z_{r_{2}^{(3 k-2)}, 2}\right) \cdots x_{n_{r_{2}^{(3 k-1)}, 2}^{(k)} \alpha_{2}}\left(z_{r_{2}^{(3 k-1)}, 2}\right) \cdots \\
& \cdots x_{n_{r_{2}^{(k), 2}}^{(k)} \alpha_{2}}\left(z_{r_{2}^{(3 k), 2}}\right) \cdots \\
& \cdots x_{n_{1,2}^{(k)} \alpha_{2}}\left(z_{1,2}\right) x_{n_{r_{1}^{(k)}, 1}^{(k)} \alpha_{1}}\left(z_{r_{1}^{(k)}, 1}\right) \cdots x_{n_{1,1}^{(k)} \alpha_{1}}\left(z_{1,1}\right) v_{L\left(\Lambda_{0}\right)} \\
& \otimes \cdots \otimes \\
& \otimes x_{n_{r_{2}^{(1)}, 2}^{(1)} \alpha_{2}}\left(z_{r_{2}^{(1)}, 2}\right) \cdots x_{n_{r_{2}^{(1)}, 2}^{(1)} \alpha_{2}}\left(z_{r_{2}^{(2)}, 2}\right) \cdots x_{n_{r_{2}^{(1)}, 2}^{(1)} \alpha_{2}}\left(z_{r_{2}^{(3)}, 2}\right) \cdots \\
& \cdots x_{n_{1,2}^{(1)} \alpha_{2}}\left(z_{1,2}\right) \cdots x_{n_{r_{1}^{(1)}, 1}^{(1)} \alpha_{1}}\left(z_{r_{1}^{(1)}, 1}\right) \cdots x_{n_{1,1}^{(1)} \alpha_{1}}\left(z_{1,1}\right) v_{L\left(\Lambda_{0}\right)} \text {, }
\end{aligned}
$$

where $\mathrm{C} \in \mathbb{C}^{*}$,

$$
0 \leq n_{p, 2}^{(t)} \leq 3, \quad n_{p, 2}^{(1)} \geq n_{p, 2}^{(2)} \geq \ldots \geq n_{p, 2}^{(k-1)} \geq n_{p, 2}^{(k)}, n_{p, 2}=\sum_{t=1}^{k} n_{p, 2}^{(t)},
$$

for every every $p, 1 \leq p \leq r_{2}^{(1)}$, so that at most one $n_{p, 2}^{(t)}(1 \leq t \leq k)$ can be 1 or 2 and

$$
0 \leq n_{p, 1}^{(t)} \leq 1, \quad 1 \leq t \leq k, n_{p, 1}^{(1)} \geq n_{p, 1}^{(2)} \geq \ldots \geq n_{p, 1}^{(k-1)} \geq n_{p, 1}^{(k)}, n_{p, 1}=\sum_{t=1}^{k} n_{p, 1}^{(t)}
$$

for every every $p, 1 \leq p \leq r_{1}^{(1)}$.

EXAMPLE 3.4. In the case when $k=2$ the projection $\pi_{\mathfrak{R}}$, where $\mathfrak{R}=$ $(6,5,4,3,2,1 ; 3,2)$, of generating function

$$
\begin{gathered}
x_{\alpha_{2}}\left(z_{6,2}\right) x_{2 \alpha_{2}}\left(z_{5,2}\right) x_{3 \alpha_{2}}\left(z_{4,2}\right) x_{4 \alpha_{2}}\left(z_{3,2}\right) x_{5 \alpha_{2}}\left(z_{2,2}\right) \\
x_{6 \alpha_{2}}\left(z_{1,2}\right) x_{\alpha_{1}}\left(z_{3,1}\right) x_{2 \alpha_{1}}\left(z_{2,1}\right) x_{2 \alpha_{1}}\left(z_{1,1}\right)
\end{gathered}
$$

on

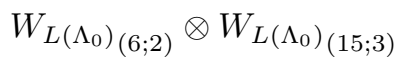

can be represented graphically as in the Figure 1, where at most one generating function of color $i=1$ is placed on every tensor factor and at most three generating functions of color $i=2$ are placed on every tensor factor.

We define the projection of monomial vector $b v_{L\left(k \Lambda_{0}\right)}$, with $b \in B_{W_{L\left(k \Lambda_{0}\right)}}$ colored with color-type $\left(r_{2}, r_{1}\right)$, charge-type $\mathfrak{R}^{\prime}$ and dual-charge-type $\mathfrak{R}$

$$
b=x_{n_{2}^{(1)}, 2} \alpha_{2}\left(m_{r_{2}^{(1)}, 2}\right) \cdots x_{n_{1,2} \alpha_{2}}\left(m_{1,2}\right) x_{n_{r_{1}^{(1)}, 1} \alpha_{1}}\left(m_{r_{1}^{(1)}, 1}\right) \cdots x_{n_{1,1} \alpha_{1}}\left(m_{1,1}\right)
$$




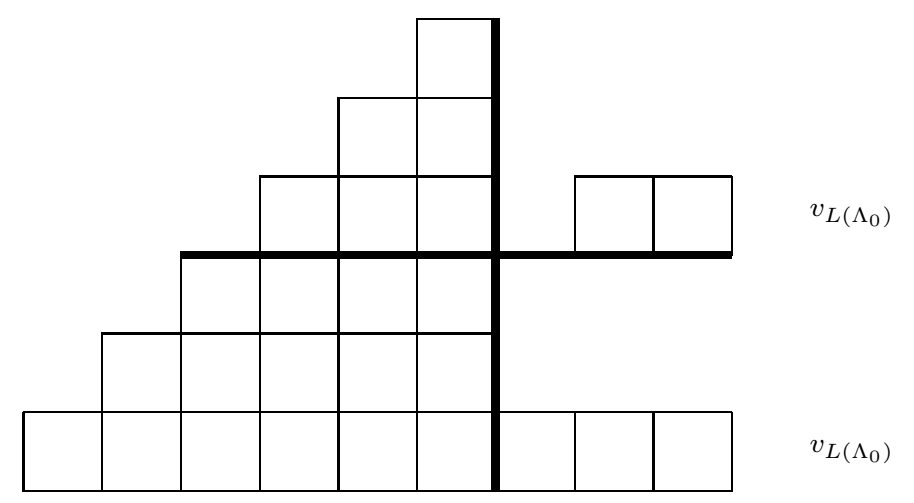

Figure 1. Example 3.4

as a coefficient of the projection of the generating function (3.11) which we denote as

$$
\pi_{\mathfrak{R}} b v_{L\left(k \Lambda_{0}\right)} .
$$

If $\bar{b} \in B_{W_{L\left(k \Lambda_{0}\right)}}$ is a monomial of charge-type $\left(\bar{n}_{\bar{r}_{2}^{(1)}, 2}, \ldots, \bar{n}_{1,2} ; \bar{n}_{\bar{r}_{1}^{(1)}, 1}, \ldots, \bar{n}_{1,1}\right)$, dual-charge-type $\overline{\mathfrak{R}}=\left(\bar{r}_{2}^{(1)}, \ldots, \bar{r}_{2}^{(3 k)} ; \bar{r}_{1}^{(1)}, \ldots, \bar{r}_{1}^{(k)}\right)$ and such that

$$
b<\bar{b},
$$

then, from the definition of projection, follows that

$$
\pi_{\mathfrak{R}} \bar{b} v_{L\left(k \Lambda_{0}\right)}=0 .
$$

We will use this property of projection $\pi_{\mathfrak{R}}$ in the proof of linear independence.

3.4. Operator $A_{\theta}$. Denote by $A_{\theta}$ the coefficient of an intertwining operator $x_{\theta}(z)$

$$
A_{\theta}=\operatorname{Res}_{z} z^{-1} x_{\theta}(z)=x_{\theta}(-1)
$$

which commutes with the action of $\mathcal{L}\left(\mathfrak{n}_{+}\right)$and such that

$$
A_{\theta} v_{L\left(\Lambda_{0}\right)}=x_{\theta}(-1) v_{L\left(\Lambda_{0}\right)} .
$$

We act with operator

$$
1 \otimes \cdots \otimes A_{\theta} \otimes \underbrace{1 \otimes \cdots \otimes 1}_{s-1 \text { factors }}, \quad s \leq k
$$

on the vector $b v_{L\left(k \Lambda_{0}\right)} \in \mathcal{B}_{W_{L\left(k \Lambda_{0}\right)}}$, where quasi-particle monomial $b$ is as in (3.12). From the definition of projection, it follows that vector

$$
\left(1 \otimes \cdots \otimes 1 \otimes A_{\theta} \otimes 1 \otimes \cdots \otimes 1\right)\left(\pi_{\mathfrak{R}} b v_{L\left(k \Lambda_{0}\right)}\right)
$$


is the coefficient of

$(3.14) \quad\left(1 \otimes \cdots \otimes A_{\theta} \otimes 1 \otimes \cdots \otimes 1\right) \pi_{\Re} x_{r_{2}^{(1)}, \alpha_{2}}\left(z_{r_{2}^{(1)}, 2}\right) \cdots x_{s \alpha_{1}}\left(z_{1,1}\right) v_{L\left(k \Lambda_{0}\right)}$.

From (3.13) it follows that in the $s$-th tensor row of (3.14) we have

$$
\begin{aligned}
& \otimes x_{n_{r_{2}^{(3)-2)}, 2}^{\left(3 s-\alpha_{2}\right.}}\left(z_{r_{2}^{(3 s-2)}, 2}\right) \cdots x_{n_{r_{2}^{(3 s-1)}, 2}^{(3)} \alpha_{2}}\left(z_{r_{2}^{(3 s-1)}, 2}\right) \cdots \\
& \cdots x_{n_{r_{2}^{(3)}, 2}^{(3 s)} \alpha_{2}}\left(z_{r_{2}^{(3 s)}, 2}\right) \cdots x_{n_{1,2}^{(s)} \alpha_{l}}\left(z_{1,2}\right) \\
& x_{n_{r_{1}^{(s)}, 1}^{(s)} \alpha_{1}}\left(z_{r_{1}^{(s)}, 1}\right) \cdots x_{\alpha_{1}}\left(z_{1,1}\right) x_{\theta}(-1) v_{L\left(\Lambda_{0}\right)} \otimes \cdots,
\end{aligned}
$$

where $0 \leq n_{p, 1}^{(s)} \leq 1$, for $1 \leq p \leq r_{1}^{(s)}$ and $0 \leq n_{p, 2}^{(s)} \leq 3$, for $1 \leq p \leq r_{2}^{(3 s-2)}$.

3.5. Operators $e_{\alpha}$. For every root $\alpha \in R$, we define on the level 1 standard module $L\left(\Lambda_{0}\right)$, the "Weyl group translation" operator $e_{\alpha}$ by

$$
\begin{aligned}
e_{\alpha}= & \exp x_{-\alpha}(1) \exp \left(-x_{\alpha}(-1)\right) \exp x_{-\alpha}(1) \exp x_{\alpha}(0) \\
& \exp \left(-x_{-\alpha}(0)\right) \exp x_{\alpha}(0),
\end{aligned}
$$

(for properly normalized root vectors, cf. [21]). Then on $L\left(\Lambda_{0}\right)$ we have

$$
\begin{gathered}
e_{\alpha} v_{L\left(\Lambda_{0}\right)}=-x_{\alpha}(-1) v_{L\left(\Lambda_{0}\right)}, \\
x_{\beta}(j) e_{\alpha}=e_{\alpha} x_{\beta}\left(j-\beta\left(\alpha^{\vee}\right)\right), \quad \beta \in R, \quad j \in \mathbb{Z} .
\end{gathered}
$$

For $\alpha=\theta$, from (3.16) and (3.17), it follows that we can (3.15) write as

$$
\begin{gathered}
\cdots \otimes x_{n_{r_{l}^{(s)-2), 2}}^{(s)} \alpha_{2}}\left(z_{r_{2}^{(3 s-2)}, 2}\right) \cdots x_{n_{r_{2}^{(3 s-1)}, 2}^{(s)} \alpha_{2}}\left(z_{r_{2}^{(3 s-1)}, 2}\right) \cdots \\
\cdots x_{n_{r_{2}^{(s)}, 2}^{(s)} \alpha_{2}}\left(z_{r_{2}^{(3 s)}, 2}\right) \cdots x_{n_{1,2}^{(s)} \alpha_{l}}\left(z_{1,2}\right) \\
x_{n_{r_{1}^{(s)}, 1}^{(k)} \alpha_{1}}\left(z_{r_{1}^{(k)}, 1}\right) z_{r_{1}^{(k)}, 1} \cdots x_{\alpha_{1}}\left(z_{1,1}\right) z_{1,1} v_{L\left(\Lambda_{0}\right)} \otimes \cdots .
\end{gathered}
$$

By taking the corresponding coefficients, we have

$\left(1 \otimes \cdots \otimes 1 \otimes A_{\theta} \otimes 1 \otimes \cdots \otimes 1\right) \pi_{\mathfrak{R}} b v_{L\left(k \Lambda_{0}\right)}=\left(1 \otimes \cdots \otimes 1 \otimes e_{\theta} \otimes 1 \otimes \cdots \otimes 1\right) \pi_{\mathfrak{R}} b^{+} v_{L\left(k \Lambda_{0}\right)}$ where

$$
b^{+}=b^{+}\left(\alpha_{2}\right) b^{+}\left(\alpha_{1}\right)=b\left(\alpha_{2}\right) x_{n_{r_{1}(1)} \alpha_{1}}\left(m_{r_{1}^{(1)}, 1}+1\right) \cdots x_{s \alpha_{1}}\left(m_{1,1}+1\right) .
$$

Now, let $\alpha=\alpha_{1}$. We consider the projection $\pi_{\mathfrak{R}} b v_{L\left(k \Lambda_{0}\right)}$ of the monomial vector $b v_{L\left(k \Lambda_{0}\right)}$ where $b \in B_{W_{L\left(k \Lambda_{0}\right)}}$ is a monomial

$$
\begin{aligned}
b= & b\left(\alpha_{2}\right) b\left(\alpha_{1}\right) x_{s \alpha_{1}}(-s) \\
= & x_{n_{2}^{(1)}, 2} \alpha_{2}\left(m_{r_{2}^{(1)}, 2}\right) \cdots x_{n_{1,2} \alpha_{2}}\left(m_{1,2}\right) \\
& x_{n_{r_{1}^{(1)}, 1} \alpha_{1}}\left(m_{r_{1}^{(1)}, 1}\right) \cdots x_{n_{2,1} \alpha_{1}}\left(m_{2,1}\right) x_{s \alpha_{1}}(-s),
\end{aligned}
$$


of dual-charge-type

$$
\mathfrak{R}=\left(r_{2}^{(1)}, \ldots, r_{2}^{(3 k)} ; r_{1}^{(1)}, \ldots, r_{1}^{(s)}, 0 \ldots, 0\right) .
$$

The projection is a coefficient of the generating function

$$
\begin{aligned}
& \pi_{\Re} x_{n_{r_{2}^{(1)}, 2} \alpha_{2}}\left(z_{r_{2}^{(1)}, 2}\right) \cdots x_{n_{1,2} \alpha_{2}}\left(z_{1,2}\right) x_{n_{r_{1}^{(1)}, 1} \alpha_{1}}\left(z_{r_{1}^{(1)}, 1}\right) \cdots x_{n_{2,1} \alpha_{1}}\left(z_{2,1}\right) \\
& \left(v_{L\left(\Lambda_{0}\right)} \otimes \cdots \otimes v_{L\left(\Lambda_{0}\right)} \otimes x_{\alpha_{1}}(-1) v_{L\left(\Lambda_{0}\right)} \otimes \cdots \otimes x_{\alpha_{1}}(-1) v_{L\left(\Lambda_{0}\right)}\right) \\
& =C x_{n_{r_{2}^{(k)-2)}, 2}^{(k)} \alpha_{2}}\left(z_{r_{2}^{(3 k-2)}, 2}\right) \cdots x_{n_{r_{2}^{(k k-1), 2}}^{(k)} \alpha_{2}}\left(z_{r_{2}^{(3 k-1)}, 2}\right) \\
& \cdots x_{n_{r_{2}^{(k), 2}(k), \alpha_{2}}}\left(z_{r_{2}^{(3 k)}, 2}\right) \cdots x_{n_{1,2}^{(k)} \alpha_{2}}\left(z_{1,2}\right) v_{L\left(\Lambda_{0}\right)} \\
& \otimes \cdots \otimes \\
& \otimes x_{n_{r_{2}^{(s)}(3 s-2), 2}^{\alpha_{2}}}\left(z_{r_{2}^{(3 s-2)}, 2}\right) \cdots x_{n_{r_{2}^{(s)}(3 s-1), 2}^{\alpha_{2}}}\left(z_{r_{2}^{(3 s-1)}, 2}\right) \\
& \cdots x_{n_{r_{2}^{(3), 2}, \alpha_{2}}^{(3 s)}}\left(z_{r_{2}^{(3 s)}, 2}\right) \cdots x_{n_{1,2}^{(s)} \alpha_{2}}\left(z_{1,2}\right) \\
& x_{n_{r_{1}^{(s)}, 1}^{(s)} \alpha_{1}}\left(z_{r_{1}^{(s)}, 1}\right) \cdots x_{n_{2,1}^{(s)} \alpha_{1}}\left(z_{2,1}\right) e_{\alpha_{1}} v_{L\left(\Lambda_{0}\right)} \\
& \otimes \cdots \otimes \\
& \otimes x_{n_{r_{2}^{(1)}, 2}^{(1)} \alpha_{2}}\left(z_{r_{2}^{(1)}, 2}\right) \cdots x_{n_{r_{2}^{(1)}, 2}^{(1)} \alpha_{2}}\left(z_{r_{2}^{(2)}, 2}\right) \cdots x_{n_{r_{2}^{(1)}, 2}^{(1)} \alpha_{2}}\left(z_{r_{2}^{(3)}, 2}\right) \\
& \cdots x_{n_{2,2}^{(1)} \alpha_{2}}\left(z_{2,2}\right) x_{n_{1,2}^{(1)} \alpha_{2}}\left(z_{1,2}\right) \\
& x_{n_{r_{1}^{(1)}, 1}^{(1)} \alpha_{1}}\left(z_{r_{1}^{(1)}, 1}\right) \cdots x_{n_{2,1}^{(1)} \alpha_{1}}\left(z_{2,1}\right) e_{\alpha_{1}} v_{L\left(\Lambda_{0}\right)} \text {. }
\end{aligned}
$$

Now, if we shift $1 \otimes \cdots \otimes e_{\alpha_{1}} \otimes e_{\alpha_{1}} \otimes \cdots \otimes e_{\alpha_{1}}$ all the way to left using commutation relations $(3.17)$ we get

$$
\pi_{\mathfrak{R}} b v_{L\left(k \Lambda_{0}\right)}=\left(1 \otimes \cdots \otimes e_{\alpha_{1}} \otimes e_{\alpha_{1}} \otimes \cdots \otimes e_{\alpha_{1}}\right) \pi_{\mathfrak{R}^{-}} b^{\prime} v_{k \Lambda_{0}}
$$

where $b^{\prime}$ is quasi-particle monomial

$$
\begin{aligned}
b^{\prime}= & b^{\prime}\left(\alpha_{2}\right) b^{\prime}\left(\alpha_{1}\right) \\
= & x_{n_{2}^{(1)}, 2} \alpha_{2}\left(m_{r_{2}^{(1)}, 2}-n_{r_{2}^{(1)}, 2}^{(1)}-\cdots-n_{r_{2}^{(1)}, 2}^{(s)}\right) \\
& \cdots x_{n_{1,2} \alpha_{2}}\left(m_{1,2}-n_{1,2}^{(1)}-\cdots-n_{1,2}^{(s)}\right) \\
& x_{n_{r_{1}^{(1)}, 1} \alpha_{1}}\left(m_{r_{1}^{(1)}, 1}+2 n_{r_{1}^{(1)}}\right) \cdots x_{n_{2,1} \alpha_{1}}\left(m_{2,1}+2 n_{1,2}\right) \\
= & x_{n_{r_{2}^{(1)}, 2} \alpha_{2}}\left(m_{r_{2}^{(1)}, 2}^{\prime}\right) \cdots x_{n_{1,2} \alpha_{2}}\left(m_{1,2}^{\prime}\right) \\
& x_{n_{r_{1}^{(1)}, 1} \alpha_{1}}\left(m_{r_{1}^{\prime(1)}, 1}^{\prime}\right) \cdots x_{n_{2,1} \alpha_{1}}\left(m_{2,1}^{\prime}\right)
\end{aligned}
$$


$0 \leq n_{p, 2}^{(t)} \leq 3, \quad 0 \leq n_{p, 1}^{(t)} \leq 1, \quad 1 \leq p \leq r_{i}^{(s)}, 1 \leq t \leq s$ of dual-charge-type

$$
\mathfrak{R}^{-}=\left(r_{2}^{(1)}, \ldots, r_{2}^{(3 s)} ; r_{1}^{(1)}-1, \ldots, r_{1}^{(s)}-1\right) .
$$

Proposition 3.5. Monomial $b^{\prime}$ (3.19) is an element of the set $B_{W_{L\left(k \Lambda_{0}\right)}}$.

Proof. We will prove that $m_{p, 2}^{\prime}, 2 \leq p \leq r_{1}^{(1)}$ and $1 \leq p \leq r_{2}^{(1)}$ satisfy the same difference conditions as energies of quasi-particle monomials from the set $B_{W_{L\left(k \Lambda_{0}\right)}}$. We will consider only energies $m_{p, 2}^{\prime}$, since for the color $i=1$ the proof is similar as in the case of $B_{2}^{(1)}$. We have two cases:

1) if $n_{p, 2} \geq 3 s$, then we have:

$$
\begin{aligned}
m_{p, 2}^{\prime} & =m_{p, 2}-3 s \\
& \leq-n_{p, 2}+\sum_{q=1}^{r_{1}^{(1)}} \min \left\{3 n_{q, 1}, n_{p, 2}\right\}-\sum_{p>p^{\prime}>0} 2 \min \left\{n_{p, 2}, n_{p^{\prime}, 2}\right\}-3 s ; \\
m_{p+1,2}^{\prime} & =m_{p+1,2}-3 s \\
& \leq m_{p, 2}-2 n_{p, 2}-3 s \\
& =m_{p, 2}^{\prime}-2 n_{p, 2} \text { when } n_{p, 2}=n_{p+1,2} ;
\end{aligned}
$$

2) if $n_{p, 2}<3 s$, then we have:

$$
\begin{aligned}
m_{p, 2}^{\prime} & =m_{p, 2}-n_{p, 2} \\
& \leq-n_{p, 2}+\sum_{q=1}^{r_{1}^{(1)}} \min \left\{3 n_{q, 1}, n_{p, 2}\right\}-\sum_{p>p^{\prime}>0} 2 \min \left\{n_{p, 2}, n_{p^{\prime}, 2}\right\}-n_{p, 2} \\
& =-n_{p, 2}+\sum_{q=2}^{r_{1}^{(1)}} \min \left\{3 n_{q, 1}, n_{p, 2}\right\}-\sum_{p>p^{\prime}>0} 2 \min \left\{n_{p, 2}, n_{p^{\prime}, 2}\right\} \\
m_{p+1,2}^{\prime} & =m_{p+1,2}-n_{p, 2} \\
& \leq m_{p, 2}-2 n_{p, 2}-n_{p, 2} \\
& =m_{p, 2}^{\prime}-2 n_{p, 2} \text { when } n_{p, 2}=n_{p+1,2} .
\end{aligned}
$$

3.6. Proof of linear independence. We prove linear independence of the set $\mathfrak{B}_{W_{L\left(k \Lambda_{0}\right)}}$ by induction on charge-type of monomials from the set $B_{W_{L\left(k \Lambda_{0}\right)}}$. Then from the Proposition 3.3 will follow $W_{L\left(k \Lambda_{0}\right)}$.

THEOREM 3.6. The set $\mathfrak{B}_{W_{L\left(k \Lambda_{0}\right)}}$ is a basis of the principal subspace 
Proof. First consider a finite linear combination

$$
\sum_{a} c_{a} b_{a} v_{L\left(k \Lambda_{0}\right)}=0
$$

of monomial vectors $b_{a} v_{L\left(k \Lambda_{0}\right)} \in \mathfrak{B}_{W_{L\left(k \Lambda_{0}\right)}}$ of the same color-type $\left(r_{2}, r_{1}\right)$. Denote by $b=b\left(\alpha_{2}\right) b\left(\alpha_{1}\right) x_{n_{1,1} \alpha_{1}}(j)$ the smallest monomial in (3.20) such that $c_{a} \neq 0$. Assume that $b$ is of charge-type

$$
\mathfrak{R}^{\prime}=\left(n_{r_{2}^{(1)}, 2}, \ldots, n_{1,2} ; n_{r_{2}^{(1)}, 1}, \ldots, n_{1,1}\right)
$$

and a dual-charge-type

$$
\mathfrak{R}=\left(r_{2}^{(1)}, \ldots, r_{2}^{(3 k)} ; r_{1}^{(1)}, \ldots, r_{1}^{\left(n_{1,1}\right)}\right),
$$

which determines the projection $\pi_{\mathfrak{R}}$ on the vector space

$$
\begin{aligned}
& W_{L\left(\Lambda_{0}\right)\left(\mu_{2}^{(k)} ; 0\right)} \otimes \cdots \otimes W_{L\left(\Lambda_{0}\right)}\left(\mu_{2}^{\left(n_{1,1}+1\right)} ; 0\right) \\
& \otimes W_{L\left(\Lambda_{0}\right)}{ }_{\left(\mu_{2}^{\left(n_{1,1}\right)} ; r_{1}^{\left(n_{1,1}\right)}\right)} \otimes \cdots \otimes W_{L\left(\Lambda_{0}\right)}\left(\mu_{2}^{(1)} ; r_{1}^{(1)}\right),
\end{aligned}
$$

where

$$
\mu_{l}^{(t)}=r_{2}^{(3 t-1)}+r_{2}^{(3 t)}+r_{2}^{(3 t-2)}, \quad 1 \leq t \leq k .
$$

$\pi_{\Re}$ maps to zero all vectors $b_{a} v_{L\left(k \Lambda_{0}\right)}$ in (3.20) with monomials $b_{a}$ of larger charge-type's than $\mathfrak{R}^{\prime}$. Now, in

$$
\sum_{a} c_{a} \pi_{\mathfrak{R}} b_{a} v_{L\left(k \Lambda_{0}\right)}=0
$$

we have a projection of $b_{a} v_{L\left(k \Lambda_{0}\right)}$, where $b_{a}$ are of charge-type $\mathfrak{R}^{\prime}$.

On $(3.22)$, we act with operators $1 \otimes \cdots \otimes A_{\theta} \otimes \underbrace{1 \otimes \cdots \otimes 1}_{n_{1,1}-1 \text { factors }}$ and commute to the left with operators $1 \otimes \cdots \otimes e_{\theta} \otimes \underbrace{1 \otimes \cdots \otimes 1}_{n_{1,1}-1 \text { factors }}$ until we get

$$
\sum_{a} c_{a} \pi_{\mathfrak{R}} b_{a}\left(\alpha_{2}\right) b_{a}\left(\alpha_{1}\right) x_{n_{1,1} \alpha_{1}}\left(-n_{1,1}\right) v_{L\left(k \Lambda_{0}\right)}=0 .
$$

Note, that in (3.23) we only have monomial vectors of charge-type $\mathfrak{R}^{\prime}$ with quasi-particle $x_{n_{1,1} \alpha_{1}}\left(-n_{1,1}\right)$, since operators used above at some point annihilate all other monomial vectors with $x_{n_{1,1} \alpha_{1}}\left(m_{1,1}\right), m_{1,1}>-j$.

From the consideration in previous subsection it follows that (3.23) can be written as

$$
1 \otimes \cdots \otimes e_{\alpha_{1}} \otimes \cdots \otimes e_{\alpha_{1}}\left(\sum_{a} c_{a} \pi_{\Re-} b_{a}^{\prime}\left(\alpha_{2}\right) b_{a}^{\prime}\left(\alpha_{1}\right) v_{L\left(k \Lambda_{0}\right)}\right)=0,
$$

and after dropping out the invertible operator $1 \otimes \cdots \otimes e_{\alpha_{1}} \otimes \cdots \otimes e_{\alpha_{1}}$ as

$$
\sum_{a} c_{a} \pi_{\mathfrak{R}^{-}} b_{a}^{\prime}\left(\alpha_{2}\right) b_{a}^{\prime}\left(\alpha_{1}\right) v_{L\left(k \Lambda_{0}\right)}=0
$$


where $b_{a}^{\prime}\left(\alpha_{2}\right) b_{a}^{\prime}\left(\alpha_{1}\right) \in B_{W_{L\left(k \Lambda_{0}\right)}}$ are quasi-particle monomials od dual-charge type

$$
\mathfrak{R}^{-}=\left(r_{2}^{(1)}, \ldots, r_{2}^{(k)} ; r_{1}^{(1)}-1, \ldots, r_{1}^{\left(n_{1,1}\right)}-1\right),
$$

with smaller charge-type from $\mathfrak{R}^{\prime}$.

We repeat the described processes, until we get

$$
\sum_{a} c_{a} \pi_{\mathfrak{R}^{-}} b_{a}\left(\alpha_{2}\right) v_{L\left(k \Lambda_{0}\right)}=0
$$

where monomial vectors $b_{a}\left(\alpha_{2}\right) v_{L\left(k \Lambda_{0}\right)}$ are colored only with color $i=2$.

Similar as in the case of $B_{2}^{(1)}$ we will see vectors $b_{a}\left(\alpha_{2}\right) v_{L\left(k \Lambda_{0}\right)}$ in $(3.25)$ as elements of

$$
\underbrace{W_{L^{A}\left(3 \Lambda_{0}\right)} \otimes \cdots \otimes W_{L^{A}\left(3 \Lambda_{0}\right)}}_{k \text { factors }}
$$

where $W_{L^{A}\left(3 \Lambda_{0}\right)}$ is the principal subspace of level 3 standard $\widetilde{s l}_{2}\left(\alpha_{2}\right)$-module $L^{A}\left(3 \Lambda_{0}\right)$ with the highest weight vector $v_{L\left(\Lambda_{0}\right)}$. Denote by $\pi_{\mathfrak{R}^{-}}^{\prime}$ the projection of (3.26) on

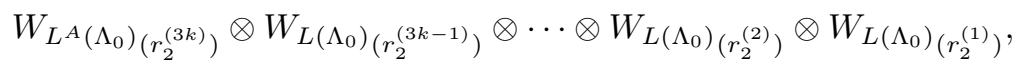

where $W_{L\left(\Lambda_{0}\right)\left(r_{2}^{(t)}\right)}, 1 \leq t \leq 3 k$ is a $\mathfrak{h}$-weighted subspace of $W_{L^{A}\left(3 \Lambda_{0}\right)}$ of weight $r_{2}^{(t)} \alpha_{2}$. From the condition (3.2), follows that monomial vectors

$$
\pi_{\mathfrak{R}^{-}}^{\prime}\left(\pi_{\mathfrak{R}^{-}} b_{a}\left(\alpha_{2}\right) v_{L\left(k \Lambda_{0}\right)}\right)
$$

are elements of vector space (3.27). Now, using Georgiev's argument from [18] follows $c_{a}=0$.

\section{Characters of PRINCIPAL SUbSPACES}

From Theorem 3.6 we easily obtain the character of the principal subspace $W_{L\left(k \Lambda_{0}\right)}$,

$$
\operatorname{ch} W_{L\left(k \Lambda_{0}\right)}:=\sum_{m, r_{1}, r_{2} \geq 0} \operatorname{dim} W_{L\left(k \Lambda_{0}\right)}{ }_{\left(m, r_{1}, r_{2}\right)} q^{m} y_{1}^{r_{1}} y_{2}^{r_{2}},
$$

where $W_{L\left(k \Lambda_{0}\right)_{\left(m, r_{1}, r_{2}\right)}}$ is a weight subspace spanned by monomial vectors of weight $-m$ and color-type $\left(r_{1}, r_{2}\right)$ (see $[4,5,18]$ ).

If we write conditions on energies of quasi-particles of a basis $\mathfrak{B}_{W_{L\left(k \Lambda_{0}\right)}}$ in terms of the dual-charge-type (and the corresponding charge-type)

$$
\left(r_{2}^{(1)}, r_{2}^{(2)}, \ldots, r_{2}^{(3 k)} ; r_{1}^{(1)}, r_{1}^{(2)}, \ldots, r_{1}^{(k)}\right):
$$




$$
\sum_{p=1}^{r_{2}^{(1)}} \sum_{q=1}^{r_{1}^{(1)}} \min \left\{n_{p, 2}, 3 n_{q, 1}\right\}=\sum_{s=1}^{k} r_{1}^{(s)}\left(r_{2}^{(2 s)}+r_{2}^{(2 s-1)}+r_{2}^{(2 s-2)}\right)
$$

$$
\sum_{p=1}^{r_{1}^{(1)}}\left(\sum_{p>p^{\prime}>0} 2 \min \left\{n_{p, 1}, n_{p^{\prime}, 1}\right\}+n_{p, 1}\right)=\sum_{s=1}^{k} r_{1}^{(s)^{2}},
$$

$$
\sum_{p=1}^{r_{2}^{(1)}}\left(\sum_{p>p^{\prime}>0} 2 \min \left\{n_{p, 2}, n_{p^{\prime}, 2}\right\}+n_{p, 2}\right)=\sum_{s=1}^{3 k} r_{2}^{(s)^{2}},
$$

then, we have the following result.

THEOREM 4.1.

$\operatorname{ch} W_{L\left(k \Lambda_{0}\right)}$

$$
=\sum_{\substack{r_{1}^{(1)} \geq \ldots \geq r_{1}^{(k)} \geq 0 \\ r_{2}^{(1)} \geq \cdots \geq r_{2}^{(3 k)} \geq 0}} \frac{q^{\left.\sum_{s=1}^{k} r_{1}^{(s)^{2}}+\sum_{s=1}^{3 k} r_{2}^{(s)}\right)^{2}-\sum_{s=1}^{k} r_{1}^{(s)}\left(r_{2}^{(3 s)}+r_{2}^{(3 s-1)}+r_{2}^{(3 s-2)}\right)}}{(q)_{r_{1}^{(1)}-r_{1}^{(2)}} \cdots(q)_{r_{1}^{(k)}}(q)_{r_{2}^{(1)}-r_{2}^{(2)}} \cdots(q)_{r_{2}^{(3 k)}}^{(3 k)}} y_{1}^{r_{1}} y_{2}^{r_{2}},
$$

where $(q)_{0}=1,(q)_{r}=(1-q)\left(1-q^{2}\right) \cdots\left(1-q^{r}\right)$ for $r>0, r_{1}=\sum_{s=1}^{k} r_{1}^{(s)}$ and $r_{2}=\sum_{s=1}^{3 k} r_{2}^{(s)}$.

At the end we state the theorem in which we describe the basis of the principal subspace $W_{N\left(k \Lambda_{0}\right)}$ :

ThEOREM 4.2. The set $\mathfrak{B}_{W_{N\left(k \Lambda_{0}\right)}}=\left\{b v_{N\left(k \Lambda_{0}\right)}: b \in B_{W_{N\left(k \Lambda_{0}\right)}}\right\}$, where

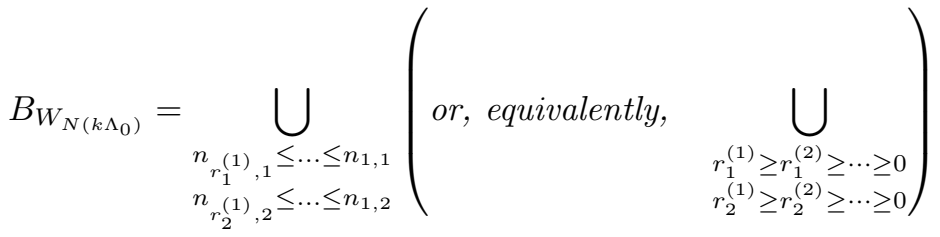

$$
\begin{aligned}
& \left\{b=b\left(\alpha_{2}\right) b\left(\alpha_{1}\right)\right. \\
& =x_{n_{r_{2}^{(1)}, 2} \alpha_{2}}\left(m_{r_{2}^{(1)}, 2}\right) \cdots x_{n_{1,2} \alpha_{2}}\left(m_{1,2}\right) x_{n_{r_{1}^{(1)}, \alpha_{1}}}\left(m_{r_{1}^{(1)}, 1}\right) \cdots x_{n_{1,1} \alpha_{1}}\left(m_{1,1}\right): \\
& m_{p, 1} \leq-n_{p, 1}-\sum_{p>p^{\prime}>0} 2 \min \left\{n_{p, 1}, n_{p^{\prime}, 1}\right\}, 1 \leq p \leq r_{1}^{(1)} \text {; } \\
& m_{p+1,1} \leq m_{p, 1}-2 n_{p, 1} \text { if } n_{p+1,1}=n_{p, 1}, 1 \leq p \leq r_{1}^{(1)}-1 \text {; } \\
& m_{p, 2} \leq-n_{p, 2}+\sum_{q=1}^{r_{1}^{(1)}} \min \left\{3 n_{q, 1}, n_{p, 2}\right\}-\sum_{p>p^{\prime}>0} 2 \min \left\{n_{p, 2}, n_{p^{\prime}, 2}\right\} \text {, } \\
& 1 \leq p \leq r_{2}^{(1)} \\
& m_{p+1,2} \leq m_{p, 2}-2 n_{p, 2} \text { if } n_{p, 2}=n_{p+1,2}, 1 \leq p \leq r_{2}^{(1)}-1
\end{aligned}
$$

is a basis of the principal subspace $W_{N\left(k \Lambda_{0}\right)}$. 
The proof of the Theorem 4.2 is similar as in the case of $W_{L\left(k \Lambda_{0}\right)}$ (see [4]), from which we can as before obtain the character of $W_{N\left(k \Lambda_{0}\right)}$ :

THEOREM 4.3.

$\operatorname{ch} W_{N\left(k \Lambda_{0}\right)}$

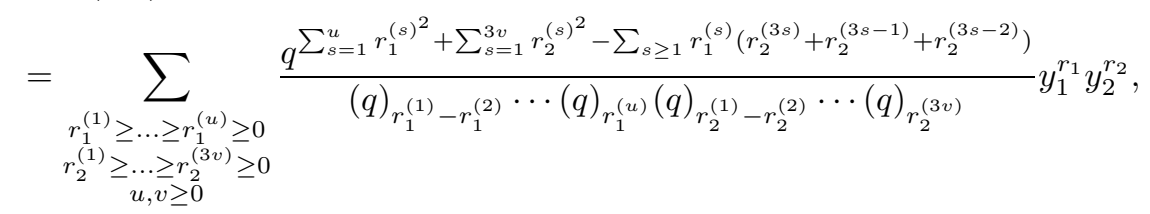

where $r_{1}=\sum_{s=1}^{u} r_{1}^{(s)}$ and $r_{2}=\sum_{s=1}^{3 v} r_{2}^{(s)}$.

From (2.4) and previous theorem follows a generalization of Euler-Cauchy theorem (cf. (2.2.8) and (2.2.9) in [1] and (4.1) in [2]):

THEOREM 4.4.

$$
\begin{aligned}
& \prod_{m>0} \frac{1}{\left(1-q^{m} y_{1}\right)} \frac{1}{\left(1-q^{m} y_{2}\right)} \frac{1}{\left(1-q^{m} y_{1} y_{2}\right)} \\
& \frac{1}{\left(1-q^{m} y_{1} y_{2}^{2}\right)} \frac{1}{\left(1-q^{m} y_{1} y_{2}^{3}\right)} \frac{1}{\left(1-q^{m} y_{1}^{2} y_{2}^{3}\right)} \\
& =\sum_{\substack{r_{1}^{(1)} \geq r_{1}^{(2)} \geq r_{1}^{(3)} \geq \ldots \geq 0 \\
r_{2}^{(1)} \geq r_{2}^{(2)} \geq r_{2}^{(3)} \geq \ldots \geq 0}} \frac{q^{\sum_{s \geq 1} r_{1}^{(s)^{2}}+\sum_{s \geq 1} r_{2}^{(s)^{2}}-\sum_{s \geq 1} r_{1}^{(s)}\left(r_{2}^{(3 s)}+r_{2}^{(3 s-1)}+r_{2}^{(3 s-2)}\right)}}{(q)_{r_{1}^{(1)}-r_{1}^{(2)}}(q)_{r_{1}^{(2)}-r_{1}^{(3)}} \cdots(q)_{r_{2}^{(1)}-r_{2}^{(2)}}(q)_{r_{2}^{(2)}-r_{2}^{(3)}} \cdots} y_{1}^{r_{1}} y_{2}^{r_{2}},
\end{aligned}
$$

where $r_{1}=\sum_{s>1} r_{1}^{(s)}$ and $r_{2}=\sum_{s>1} r_{2}^{(s)}$. The sum on the right side of (4.5) is over all descending infinite sequences of non-negative integers with finite support.

\section{ACKNOWLEDGEMENTS}

I am very grateful to Mirko Primc for his help and valuable suggestions during the preparation of this work. I would like to thank to Dražen Adamović for supporting my research. I also thank the referee for pointing out that Theorem 4.4 (Theorem 1.2 in the Introduction) is not of Rogers-Ramanujan type, but rather a generalization of Euler-Cauchy identity.

\section{REFERENCES}

[1] G. E. Andrews, The theory of partitions, Encyclopedia of Mathematics and Its Applications, Vol. 2, Addison-Wesley, 1976.

[2] G. E. Andrews, Partitions and Durfee dissection, Amer. J. Math. 101 (1979), 735742.

[3] E. Ardonne, R. Kedem and M. Stone, Fermionic characters and arbitrary highestweight integrable $\widehat{s l}_{r+1}$-modules, Comm. Math. Phys. 264 (2006), 427-464. 
[4] M. Butorac, Combinatorial bases of principal subspaces for the affine Lie algebra of type $B_{2}^{(1)}$, J. Pure Appl. Algebra 218 (2014), 424-447.

[5] M. Butorac, Quasi-particle bases of principal subspaces for the affine Lie algebras of type $B_{l}^{(1)}$ and $C_{l}^{(1)}$, Glas. Mat. Ser. III 51(71) (2016), 59-108.

[6] C. Calinescu, Intertwining vertex operators and certain representations of $\widehat{s l(n)}$, Commun. Contemp. Math. 10 (2008), 47-79.

[7] C. Calinescu, Principal subspaces of higher-level standard $\widehat{s l(3)}$-modules, J. Pure Appl. Algebra 210 (2007), 559-575.

[8] C. Calinescu, J. Lepowsky and A. Milas, Vertex-algebraic structure of the principal subspaces of certain $A_{1}^{(1)}$-modules, I. Level one case, Internat. J. Math. 19 (2008), $71-92$.

[9] C. Calinescu, J. Lepowsky and A. Milas, Vertex-algebraic structure of the principal subspaces of certain $A_{1}^{(1)}$-modules, II. Higher-level case, J. Pure Appl. Algebra 212 (2008), 1928-1950.

[10] C. Calinescu, J. Lepowsky and A. Milas, Vertex-algebraic structure of the principal subspaces of level one modules for the untwisted affine Lie algebras of types $A, D, E$, J. Algebra 323 (2010), 167-192.

[11] C. Calinescu, J. Lepowsky and A. Milas, Vertex-algebraic structure of principal subspaces of standard $A_{2}^{(2)}$-modules, I, Internat. J. Math. 25 (2014), 1450063, $44 \mathrm{pp}$

[12] C. Calinescu, A. Milas and M. Penn, Vertex algebraic structure of principal subspaces of basic $A_{2 n}^{(2)}$-modules, J. Pure Appl. Algebra 220 (2016), 1752-1784.

[13] S. Capparelli, J. Lepowsky and A. Milas, The Rogers-Ramanujan recursion and intertwining operators, Commun. Contemp. Math. 5 (2003), 947-966.

[14] S. Capparelli, J. Lepowsky and A. Milas, The Rogers-Selberg recursions, the GordonAndrews identities and intertwining operators, Ramanujan J. 12 (2006), 379-397.

[15] E. Feigin, The PBW filtration, Represent. Theory 13 (2009), 165-181.

[16] B. L. Feigin and A. V. Stoyanovsky, Functional models of the representations of current algebras, and semi-infinite Schubert cells, (Russian) Funktsional. Anal. i Prilozhen. 28 (1994), 68-90, 96; translation in Funct. Anal. Appl. 28 (1994), 5572.

[17] I. B. Frenkel, Y.-Z. Huang and J. Lepowsky, On axiomatic approaches to vertex operator algebras and modules, Memoirs of the Amer. Math. Soc. 104, (1993), no. 494, $64 \mathrm{pp}$.

[18] G. Georgiev, Combinatorial constructions of modules for infinite-dimensional Lie algebras, I. Principal subspace, J. Pure Appl. Algebra 112 (1996), 247-286.

[19] D. Gepner, New conformal theories associated with Lie algebras and their partition functions, Nuclear Phys. B 290 (1987), 10-24.

[20] M. Jerković and M. Primc, Quasi-particle fermionic formulas for $(k, 3)$-admissible configurations, Cent. Eur. J. Math. 10 (2012), 703-721.

[21] V. G. Kac, Infinite dimensional Lie algebras, 3rd ed., Cambridge University Press, Cambridge, 1990.

[22] S. Kožić, Principal subspaces for quantum affine algebra $U_{q}\left(A_{n}^{(1)}\right)$, J. Pure Appl. Algebra 218 (2014), 2119-2148.

[23] S. Kožić, Vertex operators and principal subspaces of level one for $U_{q}\left(\widehat{\mathfrak{s t}_{2}}\right)$, J. Algebra 455 (2016), 251-290.

[24] S. Kožić and M. Primc, Quasi-particles in the principal picture of $\widehat{\mathfrak{s l}_{2}}$ and RogersRamanujan-type identities, preprint.

[25] J. Lepowsky and H.-S. Li, Introduction to vertex operator algebras and their representations, Birkhäuser, Boston, 2003. 
[26] J. Lepowsky and M. Primc, Standard modules for type one affine Lie algebras, Lecture Notes in Math. 1052 (1984), 194-251.

[27] A. Meurman and M. Primc, Annihilating fields of standard modules of $\widetilde{s l}(2, \mathbb{C})$ and combinatorial identities, Mem. Amer. Math. Soc. 137 (1999), no. 652, 89pp.

[28] M. Penn and C. Sadowski, Vertex-algebraic structure of principal subspaces of basic $D_{4}^{(3)}$-modules, The Ramanujan Journal, to appear.

[29] M. Penn and C. Sadowski, Vertex-algebraic structure of principal subspaces of basic modules for twisted affine Kac-Moody Lie algebras of type $A_{2 n+1}^{(2)}, D_{n}^{(2)}, E_{6}^{(2)}$, preprint.

[30] C. Sadowski, Presentations of the principal subspaces of the higher-level standard $\widehat{\mathfrak{s l}(3)}$-modules, J. Pure Appl. Algebra 219 (2015), 2300-2345.

[31] C. Sadowski, Principal subspaces of higher-level standard $\widehat{\mathfrak{s l}(n)}$-modules, Int. J. Math. 26 (2015).

M. Butorac

Department of Mathematics

University of Rijeka

Radmile Matejčić 2, 51000 Rijeka

Croatia

E-mail: mbutorac@math.uniri.hr

Received: 22.3.2016. 